


\title{
BRIOFLORA DO ESTADO DO PIAUÍ: NOVOS REGISTROS PARA A CAATINGA E CERRADO
}

Amanda Caroline Rodrigues da Silva ${ }^{1}$, Hermeson Cassiano de Oliveira², Gonçalo Mendes da Conceição ${ }^{3}$.

1 Mestranda - Programa de Pós-Graduação em Biodiversidade, Ambiente e Saúde/PPGBAS, Universidade Estadual do Maranhão/UEMA - Centro de Estudos Superiores de Caxias/CESC, Caxias, 65604-380, MA, Brasil. E-mail: amandacrds@hotmail.com.

2 Professor Doutor do Departamento de Biologia da Universidade Estadual do Piauí/UESPI, Campus Heróis do Jenipapo, Campo Maior, PI, Brasil.

3 Professor Doutor do Departamento de Biologia, da Universidade Estadual do Maranhão/UEMA, Centro de Estudos Superiores de Caxias/CESC.

Recebido em: 06/04/2019 - Aprovado em: 10/06/2019 - Publicado em: 30/06/2019 DOI: 10.18677/EnciBio_2019A139

RESUMO

Os biomas Caatinga e Cerrado, são os menos estudados em relação à brioflora, quando comparados com as Florestas Atlântica e Amazônica. O estado do Piauí possui vegetação de transição ou de ecótonos e suas formações vegetais sofrem a influência de ambos os biomas, Caatinga e Cerrado. O Objetivo do estudo foi inventariar as espécies de musgos e hepáticas que ocorrem no estado do Piauí e os biomas nos quais estão inseridas. Foram encontradas 56 espécies de briófitas, 18 famílias e 30 gêneros. Destas, nove são hepáticas (Marchanthyophyta) e 47 são musgos (Bryophyta). A distribuição por bioma mostrou que o Cerrado apresentou o maior número de novos registros, com nove espécies, e Caatinga com sete. Das espécies encontradas, 41 correspondem novos registros para o Piauí e uma nova ocorrência para o Nordeste brasileiro. O substrato do tipo rupícola foi o mais colonizado, com 47 espécies. Os resultados obtidos representam uma importante contribuição para o conhecimento da flora briofítica do Estado, uma vez que amplia consideravelmente os dados da distribuição geográfica das espécies no Cerrado e Caatinga.

PALAVRAS-CHAVE: Florística, hepáticas, musgos.

\section{BRIOFLORA OF THE STATE OF PIAUÍ: NEW REGISTROS FOR THE CAATINGA AND CERRADO}

\begin{abstract}
The Caatinga and Cerrado biomes are the least studied in relation to the bryoflora, when compared to the Atlantic and Amazonian Forests. The state of Piauí has transitional or ecotone vegetation and its plant formations are influenced by both biomes, Caatinga and Cerrado. The aim of this paper was to inventory the moss and hepatic species that occur in the state of Piauí and verify the biomes in which they are inserted. There were 56 species of bryophytes, 18 families and 30 genera. Of ENCICLOPÉDIA BIOSFERA, Centro Científico Conhecer - Goiânia, v.16 n.29; p. 1809 2019
\end{abstract}


these, nine are hepatic (Marchanthyophyta) and 47 are mosses (Bryophyta). The distribution by biome showed that the Cerrado had the highest number of new records with nine species, and Caatinga with seven. Of the species found, 41 correspond to new records for the state of Piauí and a new occurrence for the Northeast region. The substrate of the rock type was the most colonized, with 47 species. The results obtained represent an important contribution to the knowledge of the Piauí bryophytic flora, since it considerably increases the data regarding the geographic distribution of many of these species and the distribution of these species in the Cerrado and Caatinga biomes

KEYWORDS: Floristic, mosses, liverworts.

\section{INTRODUÇÃO}

As briófitas são representadas por três divisões: Bryophyta/musgos, Marchantiophyta/hepáticas e Anthocerotophyta/antóceros (GOFFINET et al., 2009). Caracterizam-se pelo pequeno porte, ausência de vasos condutores lignificados, apresentando em seu ciclo de vida um esporófito de vida curta e nutricionalmente dependente do gametófito, que é perene e dominante, necessitando de água para a reprodução sexuada (CRANDALL-STOLLER et al., 2009).

As briófitas desempenham um relevante papel ecológico para os ecossistemas, tais como: importante componente da biomassa global, ciclagem de carbono e nutrientes através do crescimento e decomposição, promovem a formação do solo, acelerando o desgaste físico-químico e aprisionando material orgânico e inorgânico. São plantas pioneiras, possibilitando o estabelecimento de outras plantas e servindo de micro-habitat para pequenos organismos e são biondicadoras da qualidade do ar e da água (GLIME, 2017).

As briófitas são amplamente distribuídas e ocorrem em todos os continentes, sendo encontradas principalmente nas áreas tropicais e com maior umidade. No mundo, são reconhecidas cerca de 18.150 espécies, distribuídas em: 150 espécies de Anthocerotophyta, 5.000 espécies de Marchantiophyta e 13.000 de Bryophyta (VANDERPOORTEN; GOFFINET, 2009). Já para o Brasil, estima-se um total de 1.524 espécies, sendo 880 espécies de musgos, 11 espécies de antóceros e 633 de hepáticas (COSTA; PERALTA, 2015).

A região Nordeste do Brasil apresenta uma diversidade de 689 espécies, ocupando a terceira posição entre as regiões do país. Para o estado do Piauí, são registradas apenas 50 espécies (COSTA; PERALTA, 2015). Dentre as poucas publicações existentes na literatura com registros para o Piauí, existem os catálogos de Yano (1989, 1995, 2005) e o trabalho de Castro et al. (2002), o qual registrou 22 espécies de musgos para o Parque Nacional de Sete Cidades.

O estado do Piauí está situado em uma área de tensão ecológica, com vegetação de transição ou de ecótonos e suas formações vegetais sofrem a influência de diferentes biomas como o Cerrado e Caatinga. Devido à elevada heterogeneidade espacial e ambiental, a cobertura vegetal do Piauí apresenta-se como um mosaico de tipos vegetacionais que vão desde os mais secos, como as Caatingas, até os mais úmidos, como as florestas estacionais semideciduais nos limites dos estados do Piauí e Maranhão (CEPRO, 1996; BOTREL et al., 2015; SOUSA, et al., 2017).O objetivo do estudo foi inventariar espécies de briófitas que ocorrem no estado do Piauí e dessa forma, contribuir com os estudos desse grupo de plantas, fornecendo dados sobre sua distribuição geográfica. 


\section{MATERIAL E MÉTODOS}

O estudo foi realizado em áreas de Caatinga, Cerrado e transicionais na Ecorregião do Complexo de Campo Maior e municípios adjacentes, localizados integralmente no estado do Piaú. A região apresenta clima quente e úmido, tropical chuvoso, com precipitação média anual de $1.300 \mathrm{~mm}$, concentrada em poucos meses entre dezembro e junho. Normalmente, apresenta mais de seis meses secos por ano. (CEPRO, 1996; SILVA et. al., 2018).

As coletas foram realizadas em diferentes regiões do Estado do Piauí (Figura 1): Cachoeira Campeira (05 19' 59" S- 42ㅇ 25' 94" W) localizada no município de Alto Longá; Cachoeira Riachão no Parque Nacional de Sete Cidades $\left(04^{\circ} 5^{\prime}\right.$ e $04^{\circ}$ $15^{\prime}$ - S e $41^{\circ} 30^{\prime}$ e $41^{\circ}$ e $45^{\prime}-$ - W) no município de Piracuruca; Serra de Santo Antônio (045' $46^{\prime \prime}$ e e $\left.41^{\circ} 11^{\prime} 41^{\prime \prime} W\right)$ no município de Campo Maior; Cachoeira do

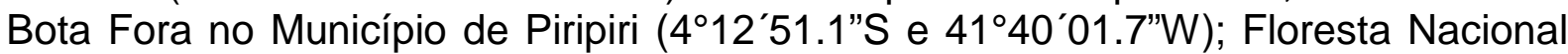
dos Palmares em Altos (S $05^{\circ} 02^{\prime}-\mathrm{W} 42^{\circ} 27^{\prime}$ ); Cachoeira de Pedra Negra no


Município de Pedro II ( 4ํ3' 62” S - 41ํ46' 26').


FIGURA 1: Mapa de localização das áreas de estudo. (1) Parque Nacional de Sete Cidades; (2) Cachoeira do Bota Fora; (3) Cachoeira do Urubu Rei; (4) Floresta Nacional dos Palmares; (5) Cachoeira da Campeira (6) Serra de Santo Antônio; (7) Cachoeira da Pedra Negra. Fonte: Mapa construído com o software SimpleMappr.

Foram analisadas amostras coletadas por diferentes coletores, as quais estão depositadas no Herbário de Criptógamas, da Universidade Estadual do Piauí (HUESPI), Campus Heróis do Jenipapo, localizado no município de Campo Maior, Piauí. Para a identificação do material, foram utilizadas as seguintes bibliografias: Gradstein e Costa (2003), Bordin e Yano (2013) e Sharp et al. (1994). A classificação adotada foi a proposta por Crandall-Stotler et al. (2009) para a as hepáticas e Goffinet et al. (2009) para os musgos. Para as diferentes áreas amostradas no estado do Piaú, as devidas autorizações de coleta e transporte de material botânico foram solicitadas ao órgão competente.

Para a distribuição geográfica das espécies, foi feita consulta à plataforma online Flora do Brasil 2020. Após o processo de herborização e identificação, duplicatas foram enviadas ao Herbário Aluízio Bittencourt (HABIT), do Centro de Estudos Superiores de Caxias/UEMA. 


\section{RESULTADOS E DISCUSSÃO}

Foram identificadas 56 espécies de briófitas, 18 famílias e 30 gêneros. Destas, nove são hepáticas (Marchanthyophyta), distribuídas em seis famílias e seis gêneros. Para a divisão Bryophyta (Musgos), foram registradas 47 espécies, 12 famílias e 24 gêneros. A distribuição por bioma mostrou que o Cerrado foi o que apresentou o maior número de novos registros, com nove espécies, seguido da Caatinga com sete espécies. Destas espécies, 41 correspondem novas ocorrências para o estado do Piauí e uma espécie para a região Nordeste (Quadro 1).

QUADRO 1. Lista de espécies de briófitas encontradas em alguns munícipios da região Norte do Piauí, por tipo de substrato, bioma e distribuição no Brasil. Tipo de substrato: Ru = rupícola, $\mathrm{Co}=$ corticícola, $\mathrm{Te}=$ terrícolo, $\mathrm{Ca}=$ casmófito, $\mathrm{Ex}=\mathrm{Epíxilo,} \mathrm{Ef}=\mathrm{Ep}$ ífila; Domínios fitogeográficos: $\mathrm{AM}=$ Amazônica, $\mathrm{CA}=$ Caatinga, $\mathrm{CE}=$ Cerrado, $\mathrm{MA}=$ Mata atlântica, $\mathrm{PN}=$ Pantanal, PM: Pampa. $\left({ }^{*}\right)$ Novas ocorrências para o Piaú́; $\left({ }^{* *}\right)$ Novas ocorrências para a região Nordeste.

\begin{tabular}{|c|c|c|c|}
\hline $\begin{array}{c}\text { Táxons } \\
\text { (Divisão/Família/Espécie) }\end{array}$ & Substrato & $\begin{array}{c}\text { Distribuição } \\
\text { Geográfica no } \\
\text { Brasil }\end{array}$ & $\begin{array}{l}\text { Biomas de } \\
\text { ocorrência }\end{array}$ \\
\hline \multicolumn{4}{|l|}{ MARCHANTIOPHYTA } \\
\hline \multicolumn{4}{|l|}{ CEPHALOZIELLACEAE Douin } \\
\hline${ }^{*}$ Cylindrocolea rhizantha (Mont.) R.M.Schust & TE & $\begin{array}{l}\text { AC, BA, GO, ES, } \\
\text { PE, RJ, SP }\end{array}$ & $\mathrm{AM}, \mathrm{CE}, \mathrm{MA}$ \\
\hline${ }^{*}$ Cylindrocolea planifolia (Steph.) R.M.Schust. & TE & $\begin{array}{l}\text { AC, BA, MG, RJ, } \\
\text { RO, SC, SP }\end{array}$ & $\mathrm{AM}, \mathrm{MA}, \mathrm{CE} \mathrm{E}^{*}$ \\
\hline \multicolumn{4}{|l|}{ FOSSOBRONIACEAE HazsI. } \\
\hline Fossombronia porphyrorhiza (Nees) Prosk & $R U$ & $\begin{array}{l}B A, C E, \quad D F, \quad E S \text {, } \\
\text { GO, MA, MG, }\end{array}$ & CA, CE, MA \\
\hline \multicolumn{4}{|l|}{ CALYPOGEIACEAE Arnell } \\
\hline${ }^{\star *}$ Calypogeia grandistipula (Steph.) Steph & $\mathrm{RU}$ & $\begin{array}{l}\text { MS, MT, PE, PI, } \\
\text { RJ, SP, TO, SP, } \\
\text { RJ, MG, ES, RS. }\end{array}$ & $\mathrm{MA}, \mathrm{CE} \mathrm{E}^{*}$ \\
\hline $\begin{array}{l}\text { FRULLLANIACEAE Lorch. } \\
{ }^{*} \text { Frullania ericoides (Nees) Mont. }\end{array}$ & $\mathrm{RU}$ & $\begin{array}{l}\text { AM, SE, AC, RJ, } \\
\text { MG, MT, PR, PB, } \\
P E, M A, ~ R S, ~ S C, \\
\text { SP, BA, CE, GO, } \\
\text { DF, ES, MS. }\end{array}$ & $\begin{array}{l}\text { AM, CA, CE, } \\
\text { MA, PN }\end{array}$ \\
\hline${ }^{*}$ Frullania gibbosa Nees & $R U$ & $\begin{array}{l}\text { AC, AM, BA, DF, } \\
E S, G O, M G, M S \\
M T, P A, P B, P E \\
R J, R R, S C, S P\end{array}$ & $\begin{array}{c}\text { AM, CA, CE, } \\
\text { MA }\end{array}$ \\
\hline \multicolumn{4}{|l|}{ LEJEUNEACEAE Cas.-Gil } \\
\hline $\begin{array}{l}\text { *Acrolejeunea torulosa (Lehm. \& Lindenb.) } \\
\text { Schiffn. }\end{array}$ & $\mathrm{CO}$ & $\begin{array}{l}\text { AC, AL, AM, BA, } \\
E S, G O, M A, M G \text {, } \\
\text { MT, PA, PE, RJ, } \\
\text { RO, RR, SP }\end{array}$ & $\mathrm{AM}, \mathrm{CE}, \mathrm{MA}$ \\
\hline
\end{tabular}




\begin{tabular}{|c|c|c|c|}
\hline \multicolumn{4}{|l|}{ RICCIACEAE L. } \\
\hline${ }^{*}$ Riccia brasiliensis Schiffn. & $R U$ & $\begin{array}{l}\text { PE, PR, RS, SC, } \\
\text { SP. }\end{array}$ & MA. $C A^{*}$ \\
\hline${ }^{*}$ Riccia stenophylla Spruce & $R U$ & $\begin{array}{l}\text { BA, CE, ES, GO, } \\
\text { MA, MS, MT, PB, } \\
\text { PE, PR, RJ, SC, SP }\end{array}$ & $\begin{array}{l}\text { CA, CE, MA, } \\
\text { PN }\end{array}$ \\
\hline \multicolumn{4}{|l|}{ BRYOPHYTA } \\
\hline $\begin{array}{c}\text { ARCHIDIACEAE Schimp. } \\
\text { Archidium ohioense Schimp. ex Mull. Hal. }\end{array}$ & $\mathrm{TE} / \mathrm{RU}$ & $\begin{array}{l}\text { AL, BA, CE, PB, } \\
\text { PE, PI, RN, GO, } \\
\text { MG, } M S, M T, R S\end{array}$ & $\begin{array}{l}\text { CA, CE, MA, } \\
\text { PN, PM. }\end{array}$ \\
\hline $\begin{array}{c}\text { BARTRAMIACEAE Schwägr. } \\
{ }^{*} \text { Philonotis cernua (Wilson) Griffin \& W.R } \\
\text { Buck }\end{array}$ & $\mathrm{TE} / \mathrm{RU}$ & $\begin{array}{l}\text { CE, DF, GO, MA, } \\
\text { MG, MT, PB, SE, } \\
\text { PR, RJ, RS, SC, } \\
\text { SP }\end{array}$ & CA, CE, MA \\
\hline${ }^{*}$ Philonotis longiseta (Michx.) E.Britton & $R U$ & CE, BA. & $\mathrm{MA}, \mathrm{CE}^{*}$ \\
\hline Philonotis uncinata (Schwägr.) & $\mathrm{RU}$ & $\begin{array}{l}\text { AC, AM, AP, PA, } \\
\text { RO, TO, BA, CE, } \\
\text { PB, PE, PI, DF, } \\
\text { GO, MT, MS, ES, } \\
\text { MG, RJ, SP, RS, } \\
\text { SC }\end{array}$ & $\begin{array}{l}\text { AM, CA, CE, } \\
\text { MA, PN, PM. }\end{array}$ \\
\hline $\begin{array}{c}\text { BRYACEAE Schwagr. } \\
{ }^{*} \text { Brachymenium exile (Dozy \& Molk.) Bosch \& } \\
\text { Sande Lac }\end{array}$ & $\mathrm{RU}$ & $\begin{array}{l}\text { RJ, PE, BA, SP, } \\
\text { GO, MS. }\end{array}$ & $\mathrm{CE}, \mathrm{MA}, \mathrm{C}$ \\
\hline $\begin{array}{l}\text { *Bryum cellulare Hook. ex Schwägr. } \\
{ }^{*} \text { Bryum coronatum Schwägr. }\end{array}$ & $\begin{array}{l}\text { TE/RU } \\
\text { TE/RU }\end{array}$ & $\begin{array}{l}\text { CE, MG } \\
\text { AL, DF, GO, MG, } \\
\text { MS, MT, PB, PE, } \\
\text { RJ, RO, SE, SP, } \\
\text { TO. }\end{array}$ & $\begin{array}{l}\text { CA, CE, MA } \\
\text { CE }\end{array}$ \\
\hline *Bryum leptocladon Sull. & TE & $\mathrm{BA}, \mathrm{DF}, \mathrm{GO}, \mathrm{SP}$ & $\mathrm{CE}, \mathrm{MA}, \mathrm{CA}^{*}$ \\
\hline Bryum limbatum Müll.Hal. & RU & $\begin{array}{l}\text { DF, MS, ES, MG, } \\
\text { RJ, SP, PR, RS, } \\
\text { SC. }\end{array}$ & CE, MA \\
\hline $\begin{array}{l}\text { CALYMPERACEAE Kindb. } \\
\text { Calymperes palisotii Schwägr. }\end{array}$ & $\mathrm{CO}$ & $\begin{array}{l}\text { AL, AM, AP, BA, } \\
E S, G O, M A, M G, \\
\text { MS, PA, PB, PE, } \\
\text { PI, PR, RJ, RN, } \\
\text { RO, RR, SE, SP, } \\
\text { TO. }\end{array}$ & $\begin{array}{l}\text { AM, CA, CE, } \\
\text { MA }\end{array}$ \\
\hline Otoblepharum albidum Hedw. & $\mathrm{CO} / \mathrm{RU}$ & $\begin{array}{l}\text { AC, AL, AM, BA, } \\
\text { CE, DF, ES, GO, } \\
\text { MA, MG, MS, MT, } \\
\text { PA, PB, PE, PI, PR, } \\
\text { RJ, RN, RO, RR, } \\
\text { RS, SC, SE, SP, } \\
\text { TO. }\end{array}$ & $\begin{array}{l}\mathrm{AM}, \mathrm{CA}, \mathrm{CE} \\
\mathrm{MA}\end{array}$ \\
\hline
\end{tabular}




\begin{tabular}{|c|c|c|c|}
\hline *Syrrhopodon gaudichaudii Mont. & $\mathrm{RU}$ & $\begin{array}{l}\text { AM, AP, BA, DF, } \\
\text { ES, GO, MA, MG, } \\
\text { MS, MT, PA, PE, } \\
\text { PR, RO, RS, SC, } \\
\text { SP, TO. }\end{array}$ & $\begin{array}{c}\text { AM, CA, CE, } \\
\text { MA }\end{array}$ \\
\hline *Syrrhopodon ligulatus Mont. & RU & $\begin{array}{l}\text { AC, AM, AP, BA, } \\
\text { DF, GO, MG, MS, } \\
\text { MT, PA, PE, RJ, } \\
\text { RO, RR, SP. }\end{array}$ & $\begin{array}{l}\mathrm{AM}, \mathrm{CE}, \mathrm{MA} \\
\mathrm{CA}^{*}\end{array}$ \\
\hline $\begin{array}{l}\text { Syrrhopodon prolifer var. scaber (Mitt.) W.D. } \\
\text { Reese. }\end{array}$ & RU & $\begin{array}{l}\text { AC, AL, AM, BA, } \\
\text { CE, DF, ES, GO, } \\
\text { MG, MT, PA, PE, } \\
\text { PR, PI, RJ, RO, } \\
\text { RS, SE, SC, SP, } \\
\text { TO }\end{array}$ & $\begin{array}{l}\mathrm{AM}, \mathrm{CA}, \mathrm{CE} \\
\mathrm{MA}\end{array}$ \\
\hline $\begin{array}{l}\text { ERPODIACEAE Broth. } \\
\text { Erpodium coronatum (Hook. \& Wilson) Mitt. }\end{array}$ & $\mathrm{CO}$ & $\begin{array}{l}\text { BA, CE, DF, GO, } \\
\text { MA, MS, MT, PB, } \\
\text { PI, SP, TO. }\end{array}$ & CA, CE, MA \\
\hline $\begin{array}{l}\text { FISSIDENTACEAE Schimp. } \\
\text { Fissidens angustifolius Sull. }\end{array}$ & $\mathrm{CA} / \mathrm{TE} / \mathrm{RU}$ & $\begin{array}{l}\text { AC, AM, BA, CE, } \\
\text { GO, MA, PA, PB, } \\
\text { PE, PI, RJ, RO, RS, } \\
\text { SP. }\end{array}$ & $\begin{array}{l}\text { AM, CA, CE, } \\
\text { MA }\end{array}$ \\
\hline${ }^{*}$ Fissidens anguste-limbatus Mitt. & RU & $\begin{array}{l}\text { AC, BA, DF, GO, } \\
\text { MA, MG, MT, PR, } \\
\text { RO, RR, RS, SP, } \\
\text { TO }\end{array}$ & $\begin{array}{l}\text { AM, CA, CE, } \\
\text { MA }\end{array}$ \\
\hline${ }^{*}$ Fissidens brevipes Besch. & RU & $\begin{array}{l}\text { DF, MG, MS, MT, } \\
\text { RJ, RO, SC, SP }\end{array}$ & $\begin{array}{l}\mathrm{AM}, \mathrm{CA}^{*}, \mathrm{CE} \\
\mathrm{MA}, \mathrm{PN}\end{array}$ \\
\hline${ }^{*}$ Fissidens crispus Mont. & RU & $\begin{array}{l}\text { AM, BA, CE, ES, } \\
\text { PE, DF, GO, MG, } \\
\text { MT, PR, RJ, RO, } \\
\text { RR, RS, SC, SP, } \\
\text { TO. }\end{array}$ & $\begin{array}{l}\mathrm{AM}, \mathrm{CA}, \mathrm{CE} \\
\mathrm{MA}\end{array}$ \\
\hline${ }^{*}$ Fissidens curvatus Hornsch. & $R U$ & $\begin{array}{l}\text { ES, MG, PE, PR, } \\
\text { RJ, RS, SC, SP }\end{array}$ & $\mathrm{MA}, \mathrm{CE}^{*}$ \\
\hline *Fissidens dissitifolius Sull. & RU & $\begin{array}{l}\text { ES, MG, PE, PR, } \\
\text { RJ, RS, SC, SP BA, } \\
\text { SP, PR }\end{array}$ & $\mathrm{MA}, \mathrm{CE}^{*}, \mathrm{CA}^{*}$ \\
\hline Fissidens elegans Brid. & RU & $\begin{array}{l}\text { AC, AM, BA, CE, } \\
\text { DF, GO, MA, MG, } \\
\text { MS, MT, PA, PB, } \\
\text { PE, PI, PR, RJ, RO, } \\
\text { RS, RR, SC, SP. }\end{array}$ & $\begin{array}{l}\mathrm{AM}, \mathrm{CA}, \mathrm{CE} \\
\mathrm{MA}\end{array}$ \\
\hline${ }^{*}$ Fissidens flaccidus Mitt. & $\mathrm{RU} / \mathrm{CA}$ & $\begin{array}{l}\text { AC, AM, PA, RO, } \\
\text { TO, BA, CE, MA, } \\
\text { PB, PE, SE, DF, } \\
\text { GO, MT, MS, ES, } \\
\text { MG, RJ, SP, PR, } \\
\text { RS. }\end{array}$ & $\begin{array}{l}\text { AM, CA, CE, } \\
\text { MA, PN, PM }\end{array}$ \\
\hline Fissidens goyazensis Broth. & RU & $\begin{array}{l}\text { AM, BA, CE, DF, } \\
\text { GO, MG, PB, PE, } \\
\text { PI, RJ, SP }\end{array}$ & $\begin{array}{l}\mathrm{AM}, \mathrm{CA}, \mathrm{CE} \\
\mathrm{MA}\end{array}$ \\
\hline${ }^{\star}$ Fissidens palmatus Hedw. & TE & $\begin{array}{l}\text { AC, BA, CE, GO, } \\
\text { MA, PE, SP. }\end{array}$ & AM, CE, MA \\
\hline
\end{tabular}




\begin{tabular}{|c|c|c|c|}
\hline${ }^{*}$ Fissidens perfalcatus Broth & $\mathrm{RU}$ & $\begin{array}{l}\text { BA, ES, GO, MG, } \\
\text { PB, PE, TO }\end{array}$ & $\mathrm{CE}, \mathrm{MA}, \mathrm{CA}^{*}$ \\
\hline${ }^{*}$ Fissidens radicans Mont. & RU & $\begin{array}{l}\text { BA, DF, ES, MA, } \\
\text { MG, PA, PB, SE, } \\
\text { SP. }\end{array}$ & $\mathrm{AM}, \mathrm{CE}, \mathrm{MA}$, \\
\hline${ }^{*}$ Fissidens ramicola Broth. & RU & $\mathrm{AM}, \mathrm{BA}, \mathrm{GO}, \mathrm{SP}$ & AM, CE, MA \\
\hline${ }^{*}$ Fissidens steerei Grout & $\mathrm{RU} / \mathrm{TE} / \mathrm{CO}$ & GO, SP. & $\mathrm{MA}, \mathrm{CE}^{*}$ \\
\hline${ }^{*}$ Fissidens submarginatus Bruch & TE/RU & $\begin{array}{l}\text { AC, AM, BA, CE, } \\
\text { DF, ES, GO, MA, } \\
\text { MG, MT, PA, PE, } \\
\text { PI, RJ, RO, RS, } \\
\text { SC, SP. }\end{array}$ & $\begin{array}{l}\text { AM, CA, CE, } \\
\text { MA }\end{array}$ \\
\hline${ }^{*}$ Fissidens zollingeri Mont. & TE/RU & $\begin{array}{l}\text { AC, AL, AM, BA, } \\
\text { CE, DF, ES, GO, } \\
\text { MA, MG, MS, MT, } \\
\text { PA, PE, PR, RJ, } \\
\text { RO, RR, RS, SC, } \\
\text { SP, TO. }\end{array}$ & $\begin{array}{l}\text { AM, CA, CE, } \\
\text { MA }\end{array}$ \\
\hline \multicolumn{4}{|l|}{ HYPNACEAE Schimp. } \\
\hline $\begin{array}{c}{ }^{*} \text { Rhacopilopsis trinitensis (Müll.Hal.) E.Britton } \\
\text { \& Dixon }\end{array}$ & RU & $\begin{array}{l}\text { AL, AM, AP, BA, } \\
\text { ES, MG, MS, MT, } \\
\text { PA, PE, RR, SP }\end{array}$ & $\begin{array}{l}\text { AM, CE, MA, } \\
\text { PN }\end{array}$ \\
\hline Vesicularia vesicularis (Schwagr.) Broth. & $\mathrm{RU} / \mathrm{EX} / \mathrm{EF}$ & $\begin{array}{l}\text { AC, AM, AP, BA, } \\
\text { ES, GO, MG, MS, } \\
\text { PA, PE, PI, PR, RJ, } \\
\text { RO, RR, RS, SC, } \\
\text { SP, TO }\end{array}$ & AM, CE, MA \\
\hline \multicolumn{4}{|l|}{ LEOCOBRYACEAE Schimp. } \\
\hline $\begin{array}{l}{ }^{*} \text { Campylopus fragilis (Brid.) Bruch \& Schimp. } \\
\text { Campylopus heterostachys (Hampe) A.Jaeger }\end{array}$ & $\begin{array}{l}R U, C O \\
R U\end{array}$ & $\begin{array}{l}\text { MG, RJ } \\
\text { BA, CE, ES, GO, } \\
\text { MA, MG, MT, PE, } \\
\text { PI, PR, RJ, RS, SP }\end{array}$ & $\begin{array}{c}\text { MA, CE* } \\
\text { AM, CA, CE, } \\
\text { MA }\end{array}$ \\
\hline${ }^{*}$ Campylopus julicaulis Broth. & $R U$ & $\begin{array}{l}B A, M G, P R, R J \\
R S, S C, S P\end{array}$ & $\mathrm{MA}, \mathrm{PM}, \mathrm{CE}^{*}$ \\
\hline $\begin{array}{l}\text { PILOTRICHACEAE Kindb. } \\
{ }^{*} \text { Callicostella pallida (Hornsch.) Ångström }\end{array}$ & $R U$ & $\begin{array}{l}\text { AC, AM, AP, PA, } \\
\text { RO, RR, TO, AL, } \\
\text { BA, CE, PE, RN, } \\
\text { SE, DF, GO, MT, } \\
\text { MS, ES, MG, RJ, } \\
\text { SP, PR, RS, SC. }\end{array}$ & $\begin{array}{l}\text { AM, CE, CA, } \\
\text { MA, PN, PM. }\end{array}$ \\
\hline $\begin{array}{l}\text { POTTIACEAE Schimp } \\
{ }^{* B a r b u l a ~ i n d i c a ~(H o o k .) ~ S p r e n g . ~}\end{array}$ & $R U$ & $\begin{array}{l}\text { AC, AM, BA, DF, } \\
\text { ES, GO, MG, MS, } \\
\text { MT, PA, PE, PR, } \\
\text { RJ, RN, RS, SE, } \\
\text { SP }\end{array}$ & $\begin{array}{l}\text { AM, CA, CE, } \\
\text { MA, PN. }\end{array}$ \\
\hline Hyophilla involuta (Hook.) A. Jaeger. & $\mathrm{CA} / \mathrm{TE} / \mathrm{RU}$ & $\begin{array}{l}\text { AL, AM, BA, CE, } \\
\text { DF, ES, GO, MA, } \\
\text { MG, MS, MT, PA, } \\
\text { PB, PE, PI, PR, RJ, }\end{array}$ & $\begin{array}{l}\text { AM, CA, CE, } \\
\text { MA, PM, PN }\end{array}$ \\
\hline
\end{tabular}




\begin{tabular}{|c|c|c|c|}
\hline \\
\hline *Plaubelia sprengelii (Schwägr.) R.H.Zander & $R U$ & $\begin{array}{l}\text { RO, RR, RS, SP. } \\
\text { AC, AM, RR, BA, } \\
\text { MA, PE, GO, MT, } \\
\text { MG, RJ, SP }\end{array}$ & $\mathrm{AM}, \mathrm{CE}, \mathrm{CA}$ \\
\hline${ }^{*}$ Tortella tortuosa (Hedw.) Limpr & TE & RJ, MA & $\mathrm{MA}, \mathrm{CE}^{*}$ \\
\hline \multicolumn{4}{|l|}{ SEMATOPHYLLACEAE Broth. } \\
\hline *Acroporium caespitosum (Hedw.) W.R. Buck & $\mathrm{CO}$ & $\begin{array}{l}\text { PB, DF, MT, PR, } \\
\text { RS. }\end{array}$ & CE, MA \\
\hline $\begin{array}{c}\text { *Brittonodoxa subpinnata (Brid.) W.R. Buck, } \\
\text { P.E.A.S.Câmara \& Carv.-Silva. }\end{array}$ & RU & $\begin{array}{l}\text { RJ, PA, GO, MG, } \\
\text { PE, SP, AM, RS, } \\
\text { BA, PR, SC, AL, } \\
\text { CE, ES, MS, AC, } \\
\text { MT, RR, RO, DF, } \\
\text { TO. }\end{array}$ & MA, CE, AM \\
\hline *Sematophyllum adnatum (Michx.) Brid. & CO/EF & $\begin{array}{l}\text { AM, BA, DF, ES, } \\
\text { GO, MA, MG, MS, } \\
\text { MT, PA, RJ, RN, } \\
\text { SP, TO. }\end{array}$ & $\begin{array}{l}\text { AM, CA, CE, } \\
\text { MA }\end{array}$ \\
\hline Microcalpe subsimplex (Hedw.) W.R. Buck & TE, RU & $\begin{array}{l}\text { AC, AL, AM, AP, } \\
\text { BA, CE, DF, ES, } \\
\text { GO, MA, MG, MS, } \\
\text { MT, PA, PE, PI, } \\
\text { PR, RJ, RO, RR, } \\
\text { RS, SC, SE, SP, } \\
\text { TO. }\end{array}$ & $\mathrm{AM}, \mathrm{CA}, \mathrm{CE}$ \\
\hline $\begin{array}{l}\quad{ }^{*} \text { Vitalia galipensis (Müll.Hal.) } \\
\text { P.E.A.S.Câmara, Carv.-Silva \& W.R. Buck }\end{array}$ & RU & $\begin{array}{l}\text { PR, SP, PA, RJ, } \\
\text { DF, MG, GO, ES, } \\
\text { TO, CE, BA, MS, } \\
\text { MA, RS, SC. }\end{array}$ & MA, CE, PM \\
\hline \multicolumn{4}{|l|}{$\begin{array}{c}\text { STEREOPHYLLACEAE W.R. Buck \& } \\
\text { Ireland }\end{array}$} \\
\hline $\begin{array}{c}\text { Entodontopsis leucostega (Brid.) Buck \& } \\
\text { Ireland }\end{array}$ & $\mathrm{EX} / \mathrm{CO} / \mathrm{RU}$ & $\begin{array}{l}\text { AC, AM, BA, CE, } \\
\text { DF, GO, MA, MG, } \\
\text { MS, MT, PA, PB } \\
\text { PE, PI, RJ, RO, } \\
\text { RR, SP, TO. }\end{array}$ & $\begin{array}{l}\text { AM, MA, CA, } \\
\text { CE, PN. }\end{array}$ \\
\hline $\begin{array}{l}{ }^{*} \text { Eulacophyllum cultelliforme (Sull.) W.R.Buck } \\
\text { \& Ireland }\end{array}$ & $\mathrm{RU}$ & $\begin{array}{l}\text { AM, BA, ES, TO, } \\
\text { MG, MS, MT, PB, } \\
\text { PE, PR, RJ, SP. }\end{array}$ & $\begin{array}{l}\mathrm{AM}, \mathrm{MA}, \mathrm{CE}, \\
\mathrm{CA} \text { * }\end{array}$ \\
\hline
\end{tabular}

As Famílias mais representativas de musgos foram Fissidentaceae (15 spp), Calymperaceae (5 spp), Bryaceae (5 spp) e Semathophyllaceae (5 spp). Para as hepáticas, as famílias com maior riqueza de espécies foram Cephaloziellaceae, Frullaniaceae e Ricciaceae, com duas espécies cada uma.

Com relação aos substratos colonizados, aquele com maior riqueza de espécies foi rupícola (47 spp.), seguido por terrícola (15 spp.), corticícola (9 spp), epíxila (2spp.), epífila (2 spp.) e casmófita (2 spp). Houveram 16 espécies (28,57\%) que tiveram preferência por mais de um tipo de substrato (polis-substrato) e 40 espécies $(71,43 \%)$ que colonizaram apenas um tipo (monos-substrato). $O$ alto número de espécies pertencentes ao grupo rupícola se deve a grande 
disponibilidade de substrato rochoso e pelo fato de a maioria dos locais de coleta apresentarem cachoeiras. Corroborando com os trabalhos realizados nesses tipos de ambientes (CARMO; PERALTA, 2016; OLIVEIRA, et al. 2018c).

Fissidens angustifolius Sull., Fissidens steerei Grout, Vesicularia vesicularis (Schwägr.) Broth., Entodontopsis leucostega (Brid.) Buck \& Ireland e Hyophilla involuta (Hook.) A. Jaeger., foram coletadas em três tipos diferentes de substratos, o que demonstra grande amplitude ecológica dessas espécies e adaptação em diversos ambientes.

O número elevado de musgos neste estudo, pode estar relacionado ao fato desses apresentarem diversos atributos morfológicos e assim exibindo uma maior capacidade de tolerar a dessecação do que as hepáticas, em ambientes semiáridos (GLIME, 2017). Constatando assim com outros trabalhos realizados em outras áreas de Caatinga e Cerrado do Nordeste (COSTA et. al., 2015; RIOS et. al., 2016; VALENTE et. al., 2017; VIEIRA et. al., 2017; SILVA et. al., 2018; COSTA et. al., 2018; BATISTA et. al., 2018; OLIVEIRA et al., 2018a; OLIVEIRA et. al., 2018b).

A maioria das espécies (50) possui uma ampla distribuição geográfica no Brasil, respondendo por $89,28 \%$ das briófitas encontradas na área. São ditas espécies tipicamente generalistas por facilmente ocupar uma variedade de ambientes e por possuírem caracteres morfológicos que proporcionam a elas resistirem a condições adversas. Tais como a presença de papilas nos filídios (adaptação para ambientes secos) presença de costa (exclusivo para musgos) e pigmentos acessórios que auxiliam na proteção contra a forte radiação solar (GOFFINET et al. 2009; GLIME, 2017). Como exemplo tem-se os musgos: Syrrhopodon prolifer var. scaber (Mitt.) W.D. Reese., Otoblepharum albidum Hedw, Microcalpe subsimplex (Hedw.) W.R. Buck, Hyophilla involuta (Hook.) A. Jaeger e Entodontopsis leucostega (Brid.) Buck \& Ireland. Seis espécies (10,72 \%) tiveram sua distribuição restrita a menos de quatro estados.

Em relação à distribuição das espécies nos biomas, Philonotis uncinata (Schwägr.), Fissidens flaccidus Mitt e Callicostella pallida (Hornsch.) Ångström, estão presentes em todos os biomas brasileiros. Houve também um aumento no número de novos registros para Caatinga e Cerrado, destacando: Fissidens curvatus Hornsch., F. dissitifolius Sull, Philonotis longiseta (Michx.) E. Britton e Tortella tortuosa (Hedw.) Limpr., musgos que antes eram registrados somente para a Mata Atlântica; e as espécies de hepáticas Calypogeia grandistipula (Steph.) Steph. e Riccia brasiliensis Schiffn., também registradas apenas para a Mata Atlântica.

\section{CONCLUSÃO}

Os resultados representam uma importante contribuição para o conhecimento da flora briofítica da região, assim amplia os dados a respeito da distribuição geográfica de muitas destas espécies e a distribuição destas nos biomas Cerrado e da Caatinga, indicando sua relevância para o inventário de briófitas do estado do Piauí e para o Brasil.

\section{AGRADECIMENTOS}

Ao Programa de Pós-Graduação em Biodiversidade, Ambiente e Saúde/PPGBAS, da Universidade Estadual do Maranhão/UEMA, pelo apoio financeiro referente a bolsa concedida à primeira autora. 


\section{REFERÊNCIAS}

BATISTA, W.V.S.M.; PÔRTO, K.C. \& SANTOS, N.D. Distribution, ecology, and reproduction of bryophytes in a humid enclave in the semiarid region of northeastern Brazil. Acta Botanica Brasilica, v.32, n.2, p.303-313, 2018. Disponível em: <http://dx.doi.org/10.1590/0102-33062017abb0339>. DOI: 10.1590/0102$33062017 a b b 0339$.

BORDIN, J. \& YANO, O. Fissidentaceae (Bryophyta) do Brasil. São Paulo: Boletim do Instituto de Botânica, p. 168, 2013.

BOTREL, R. T.; BRITO, D.R.S; SOUSA, W.C.; SOUZA, A.M.; HOLANDA, A.C. Fenologia de uma espécie arbórea em ecótono Caatinga / Cerrado no sul do Piauí. Revista Verde de Agroecologia e desenvolvimento Sustentável, v.10, n.3, p.7, 2015. Disponível em: <http://dx.doi.org/10.18378/rvads.v10i3.3587>. DOI: $10.18378 /$ rvads.v10i3.3587.

CARMO, D.M; \& PERALTA, D.F. Survey of bryophytes in Serra da Canastra National Park, Minas Gerais, Brazil. Acta Botanica Brasilic, v.30, p.254-265, 2016. Disponível em: <https://doi.org/10.1590/010233062014abb3608.> DOI: 10.1590/010233062014abb3608

CASTRO, N.M.C.F.; PÔRTO, K.C.; YANO, O. \& CASTRO, A.A.J.F. Levantamento Florístico de Bryopsida de Cerrado e Mata Ripícola do Parque Nacional de Sete Cidades, Piauí, Brasil. Acta Botanica Brasilica, v.16, n.1, p.61-76, 2002. Disponível em: <http://dx.doi.org/10.1590/S0102-33062002000100008>. DOI: 10.1590/S010233062002000100008 .

CEPRO. Diagnóstico das condições ambientais do estado Piauí. Teresina: p.154, 1996.

COSTA, D.P. ; PERALTA, D.F. Bryophytes diversity in Brazil. Rodriguésia, v.66, n.4, p.1063-1071, 2015. Disponível em: <http://dx.doi.org/10.1590/21757860201566409>. DOI: 10.1590/2175-7860201566409.

COSTA, F.B.; SILVA, E.O. ; CONCEIÇÃO, G.M. Hepáticas (Marchantiophyta) e musgos (Bryophyta) da Área de Proteção Ambiental do Buriti do Meio, município de Caxias, Maranhão, Brasil. Scientia Plena, v.11, p.1-4, 2015. Disponível em: <https://www.scientiaplena.org.br/sp/article/view/1959/0>. Acesso em: 20 de Fev. 2019.

COSTA, A.M.R.; OLIVEIRA, R.R.; SANTOS-SILVA, D.L.; SÁ, N.A.S.; CONCEIÇÃO, G.M. Briófitas do Cerrado Maranhense, Nordeste do Brasil. NBC-Periódico Científico do Núcleo de Biociências, v. 8, n. 16, p.33-45, 2018. Disponível em: $<$ https://www.metodista.br/revistas/revistas-izabela/index.php/bio/article/view/1698>. Acesso em: 15 de Fev. 2019.

CRANDALL-STOTLER, B, STOTLER, R E. ; LONG, DG. Morphology and classification of the Marchantiophyta. In: GOFFINET, B.; SHAW, J.A. (eds.). Bryophyte Biology. 2 ed. New York: Cambridge University Press. P. 1-54, 2009. 
FLORA DO BRASIL 2020. Jardim Botânico do Rio de Janeiro. Disponível em:<http://floradobrasil.jbrj.gov.br/ >. Acesso em: 22 de Fev. 2019.

GLIME, J. M. Capítulo 1 - Introdução. In: Bryophyte Ecology Ebook. Michigan Technological University and the International Association of Bryologists. v.1, 2017. Disponível em: <http://www.bryoecol.mtu.edu/> Acesso em: 18 de Fev/ 2019.

GOFFINET, B.; BUCK, W.R.; SHAW, A.J. Morphology and Classification of the Bryophyta. In: Goffinet, B. \& Shaw, A.J. (eds.). Bryophyte Biology. New York: Cambridge University Press, p. 55-138. 2009.

GRADSTEIN, S.R. \& COSTA, D.P. The Hepaticae and Anthocerotae of Brazil. New Yourk: Memoirs of The New York Botanical Garden, v.87, p. 318, 2003.

OLIVEIRA, R.F.; SILVA, G.S.; OLIVEIRA, R.R.; OLIVEIRA, H.C. \& CONCEICAO, G.M. Musgos (Bryophyta) de um fragmento de cerrado maranhense, Brasil. Biota Amazônia, v.8, n.2, p.12-18, 2018a. Disponível em: <http://dx.doi.org/10.18561/21795746>. DOI: 10.18561/21795746.

OLIVEIRA, R.R.; MENDEIROS, D.L.; OLIVEIRA, H.C. \& CONCEIÇÃO, G.M. Briófitas de área sob o domínio fitogeográfico do Cerrado e novas ocorrências para 0 Maranhão e região Nordeste do Brasil. Iheringia Série Botânica, v.73, n.2, p.1-195, 2018b. Disponível em: <http://dx.doi.org/10.21826/2446-8231201873211>. DOI: 10.21826/2446-8231201873211.

OLIVEIRA, R.R.; OLIVEIRA, H.C.; PERALTA, D.F. \& CONCEIÇÃO, G.M. Acrocarpic mosses (Bryophyta) of Chapada das Mesas National Park, Maranhão, Brazil. Check List, v.14, n.6, p.967-975, 2018c. Disponível em: $<$ https://doi.org/10.15560/14.6.967>. DOI:10.15560/14.6.967.

RIOS, A. B. M.; OLIVEIRA, J. P. S.; SILVA, R. P.; OLIVEIRA-NETO, J. F. O.; OLVEIRA, L. S.; PERALTA, D. F.; MACCGNAN, D. H. B. Bryophyte diversity in area of Brazilian Cerrado in Central-West. Neotropical Biology and Conservation, v.12, n. 3, p.132-140, 2016. Disponível em: <https://dx.doi.org/10.4013/nbc.2016.113.03>. DOI: $10.4013 /$ nbc.2016.113.03.

SHARP, A.J.; CRUM, H. ; ECKEL, P. The Moss Flora of Mexico. New Yourk: Memoirs of The New York Botanical Garden, v.69, p.1-1113, 1994.

SILVA, J. B.; SFAIR, J. C.; SANTOS, N. D.; PÔRTO, K. C. Bryophyte richness of soil islands on rocky outcrops is not driven by island size or habitat heterogeneity. Acta Botanica Brasilica, v.32, n.2, 2018. Disponível em: < http://dx.doi.org/10.1590/010233062017abb0281 >. DOI: 10.1590/0102-33062017abb0281.

SILVA, D.; CASTRO, A.; ALENCAR, N.; FARIAS, R. Diagnóstico Fitossociológico e uso da vegetação de um Cerrado Ecotonal da região Setentrional do Piauí. Revista Geográfica Acadêmica, v.12, n.1, p. 76-92, 2018. Disponível em: < http://dx.doi.org/10.18227/1678-7226rga.v12i1.5003> DOI: $10.18227 / 1678-$ 7226rga.v12i1.5003.

SOUZA, M.P.; COUTINHO, J.M.C.P.; SILVA, L.S.; AMORIM, F.S. ; ALVES, A.R. Composição e estrutura da vegetação de caatinga no sul do Piauí, Brasil. Revista Verde de Agroecologia e Desenvolvimento Sustentável, v.12, n.2, p.210-217, 
2017. Disponível em:<http://dx.doi.org/10.18378/rvads.v12i2.4588>. DOI: 10.18378/rvads. v12i2.4588.

VANDERPOORTEN, A. ; GOFFINET, B. Introduction of Bryophytes. Cambridge University Press, p.294, 2009.

VALENTE, B.E.; PÔRTO, C.K. ; BASTOS, C.J.P. Habitat heterogeneity and diversity of bryophytes in campos rupestres. Acta Botanica Brasilica, v.31, n.2, p.241-249, 2017. Disponível em: <http://dx.doi.org/10.1590/0102-33062016abb0450>. DOI: 10.1590/0102-33062016abb0450.

VIEIRA, H. C. A.; OLIVEIRA, R. R.; SILVA, M. L. A.; SILVA, D. L. S.; CONCEIÇÃO, G. M.; OLIVEIRA, H. C. Briófitas de ocorrências em São João do Sóter, Maranhão, Brasil. Acta Brasiliensis, v.1, n. 2, p. 8-12, 2017. Disponível em: https: <//doi.org/10.22571/Actabra12201745> DOI: 10.22571/Actabra12201745.

YANO, O. An additional checklist of Brazilian bryophytes. Journal of the Hattori Botanical Laboratory, v.66, n.4, p.371-434, 1989. Disponível em : $<$ http://dx.dCoi.org/10.1590/2175-7860201566409>. DOI: 10.1590/21757860201566409 .

YANO, O. A new additional annotated ckecklist of Brazilian bryophytes. Journal of the Hattori Botanical Laboratory. v.78, p.137-182, 1995.

YANO, O. Novas ocorrências de Bryophyta para vários estados do Brasil. Acta Amazonica. v. 34, n.4, p.559-576, $2005 . \quad$ Disponível em: <http://dx.doi.org/10.1590/S0044-59672004000400008>. DOI: $10.1590 / S 0044-$ 59672004000400008. 